\title{
Gemcitabine Plus Cisplatin Split Versus Gemcitabine Plus Carboplatin for Advanced Urothelial Cancer With Cisplatin-unfit Renal Function
}

\author{
KOUJI IZUMI, HIROAKI IWAMOTO, HIROSHI YAEGASHI, KAZUYOSHI SHIGEHARA, \\ TAKAHIRO NOHARA, YOSHIFUMI KADONO and ATSUSHI MIZOKAMI \\ Department of Integrative Cancer Therapy and Urology, \\ Kanazawa University Graduate School of Medical Science, Kanazawa, Japan
}

\begin{abstract}
Background: Combination chemotherapy with gemcitabine and cisplatin is the standard first-line treatment for advanced urinary tract urothelial cancer. Carboplatin is often substituted for cisplatin in patients who are cisplatin-ineligible, such as those with a glomerular filtration rate less than $60 \mathrm{ml} / \mathrm{min}$. However, carboplatin-based chemotherapy has not been not confirmed as meeting the standard of care based on randomized controlled trials, and it is still unclear whether carboplatin can offer prognosis comparable to that with cisplatin. Patients and Methods: Patients with advanced urothelial cancer who underwent gemcitabine/cisplatin $(G C)$ split or gemcitabine/ carboplatin (GCarbo) for renal dysfunction with a glomerular filtration rate of approximately $40-60 \mathrm{ml} / \mathrm{min}$ between 2008 and 2015 were chosen and reviewed using their charts. Patients with normal renal function treated with $G C$ were also reviewed as a reference group. Results: A total of 41 patients, including 10 treated with GCsplit, 16 treated with GCarbo, and 15 treated with GC, were analyzed. The median overall and progression-free survival in GCsplit and GCarbo groups were 18.1 and 12.5 months $(p=0.0454)$ and 9.9 and 6.4 months ( $p=0.0404)$, respectively. Neutropenia was relatively more severe in the GCsplit group than the GCarbo group ( $p=0.0103)$. Conclusion: GCsplit may be a better treatment option for patients with advanced urothelial cancer with cisplatin-ineligible renal function. However, a prospective randomized controlled trial with a large-sized population is warranted to confirm our preliminary results.
\end{abstract}

This article is freely accessible online.

Correspondence to: Kouji Izumi, Department of Integrative Cancer Therapy and Urology, Kanazawa University Graduate School of Medical Science, 13-1 Takara-machi, Kanazawa, Ishikawa 9208641, Japan. Tel: +81 762652393, Fax: +81 762226726, e-mail: azuizu2003@yahoo.co.jp

Key Words: Carboplatin, cisplatin, renal function, urothelial cancer.
Localized upper urinary tract urothelial cancer and localized muscle-invasive bladder cancer are usually treated with nephroureterectomy and cystectomy; however, the incidence of recurrence or metastasis is not rare (1). Systemic chemotherapy, with or without metastasectomy, is required for such patients, as well as for patients with inoperable advanced urinary tract urothelial cancer (2). Platinum-based chemotherapy is the first-line treatment for inoperable locally advanced or metastatic urinary tract urothelial cancer (LAMUC) (3). Gemcitabine/cisplatin (GC) was developed as an alternative first-line treatment supported by similar clinical efficacy and less severe adverse events (AEs) compared to the conventional standard of care comprising methotrexate/ vinblastine/doxorubicin/cisplatin (4). Impaired renal function, poor performance status, advanced age, and comorbidities limit the use of cisplatin; thus, an alternative treatment protocol with carboplatin was developed $(5,6)$. However, the gemcitabine/carboplatin (GCarbo) protocol has not yet been confirmed as meeting the standard of care based on randomized controlled trials, and it is still unclear whether the carboplatin-based chemotherapy can offer a prognosis comparable to that with cisplatin-based chemotherapy. Recently, a GCsplit protocol, in which the cisplatin dose is split into 2 days (i.e. on days 2 and 3, or on days 2 and 9), was proposed for patients with LAMUC who are ineligible for cisplatin (7). However, as far as we are aware, no study has compared GCsplit and GCarbo directly in terms of survival. In this study, the effect of GCsplit protocol on survival was retrospectively compared to that using GCarbo protocol for patients with LAMUC with cisplatin-unfit renal function.

\section{Patients and Methods}

Protocols of chemotherapy. GC, GCsplit, and GCarbo were administered using previously reported protocols, briefly: GC: 1,000 $\mathrm{mg} / \mathrm{m}^{2}$ Gemcitabine (days 1,8 , and 15 ) and $70 \mathrm{mg} / \mathrm{m}^{2}$ cisplatin (day 2), every 4 weeks (4); GCsplit: $1000 \mathrm{mg} / \mathrm{m}^{2}$ gemcitabine (days 1 , 8 , and 15 ) and $35 \mathrm{mg} / \mathrm{m}^{2}$ cisplatin (days 2 and 3 or days 2 and 9), every 4 weeks; GCarbo: $1,000 \mathrm{mg} / \mathrm{m}^{2}$ Gemcitabine (days 1 and 8 ) 
and carboplatin at an area under the curve (AUC) of 4.5 (day 1), every 3 weeks (6). Chemotherapy was continued until disease progression or emergence of any severe AEs.

Study population. Patients with LAMUC who underwent GCsplit or GCarbo as a first-or second-line therapy for renal dysfunction unfit for cisplatin with a glomerular filtration rate (GFR) of approximately 40-60 ml/min between 2008 and 2015 were chosen and reviewed using their charts. Patients with normal renal function treated with GC between 2008 and 2015 were also reviewed as a reference group. Patients who underwent these chemotherapies as neoadjuvant or adjuvant treatments associated with total cystectomy or nephroureterectomy were excluded from this study.

Definition and outcomes. Application of GCsplit or GCarbo was determined for patients with 40-60 $\mathrm{ml} / \mathrm{min}$ of either estimated GFR based on the patient's serum creatinine level or 24-h creatinine clearance with correction by body surface area. Physicians assigned patients at their discretion to receive either GCsplit or GCarbo therapy and determined the timing of cisplatin injections (days 2 and 3, or days 2 and 9) and AUC of carboplatin. Patients treated with GCsplit and GCarbo were compared for background, response in tumor size, overall survival (OS), and progression-free survival (PFS) from the first of chemotherapy. The frequency and grade of hematological AEs were also investigated using Common Terminology Criteria for Adverse Events (CTCAE) v4.0 (8). The new response evaluation criteria in solid tumors, Revised RECIST guideline (version 1.1) (9), was used for response in tumor size measured by computed tomography or magnetic resonance image every 1 to 6 months.

Statistical analysis. Statistical analyses were performed using the Prism software (GraphPad, San Diego, CA, USA). Comparisons between the two groups were performed using unpaired two-sided $t$-test, Fisher exact test, and Chi-square test for trend. The KaplanMeier method was used to display OS and PFS, and the log-rank test was used for statistical analyses. In all analyses, $p$-values less than 0.05 were considered statistically significant.

\section{Results}

Patient background. A total of 10, 16, and 15 patients were treated with GCsplit, GCarbo, and GC, respectively. There were no differences in gender, age, median number of cycles administered, primary site, history of resection of primary site, and the rate of second-line use between the GCsplit and GCarbo groups. However, more men and patients with resection of primary site were included in the GCarbo group than in the GCsplit group (Table I). Although there were significant differences in serum creatinine and estimated GFR between the GCsplit and GCarbo groups, there was no significant difference in 24-h creatinine clearance corrected with body surface area between these groups (Table II, Figure 1).

Response in tumor size. Waterfall plots of the best response rate in each group are shown in Figure 2. The overall response rates (ORR), including complete response (CR) and partial response (PR), in the GCsplit, GCarbo, and GC groups were
Table I. Characteristics of patients treated with gemcitabine/cisplatin (GC), GCsplit or gemcitabine/carboplatin (GCarbo). The GCsplit and GCarbo groups did not differ significantly in these characteristics.

\begin{tabular}{|c|c|c|c|}
\hline Characteristic & $\begin{array}{l}\text { GCsplit } \\
(\mathrm{n}=10)\end{array}$ & $\begin{array}{c}\text { GCarbo* } \\
(\mathrm{n}=16)\end{array}$ & $\begin{array}{c}\text { GC } \\
(\mathrm{n}=15)\end{array}$ \\
\hline \multicolumn{4}{|l|}{ Gender } \\
\hline Male & 4 & 12 & 9 \\
\hline Female & 6 & 4 & 6 \\
\hline \multicolumn{4}{|l|}{ Age, years } \\
\hline Median (range) & $73(62-78)$ & $76(64-83)$ & $71(54-82)$ \\
\hline \multicolumn{4}{|l|}{ No. of courses } \\
\hline Median (range) & $3(1-5)$ & $3(1-6)$ & $3(1-5)$ \\
\hline \multicolumn{4}{|l|}{ Primary site } \\
\hline Bladder & 6 & 6 & 10 \\
\hline UUT & 4 & 10 & 5 \\
\hline \multicolumn{4}{|l|}{ Surgery } \\
\hline Yes & 3 & 9 & 3 \\
\hline No & 7 & 7 & 12 \\
\hline \multicolumn{4}{|l|}{ Second-line } \\
\hline Yes & 6 & 8 & 8 \\
\hline No & 4 & 8 & 7 \\
\hline
\end{tabular}

*Median area under the curve was 5 (range=3-5). UUT: Upper urinary tract.

$80 \%, 56 \%$, and $67 \%$, respectively (not significant, Table III). CR in the GCsplit, GCarbo, and GC groups was $20 \%, 19 \%$, and $33 \%$, respectively. In CR cases in each group, relatively small tumors completely disappeared in both the GCarbo and GC groups. Interestingly, an invasive huge mass of left renal pelvic cancer in a case of the GCsplit group also completely disappeared (Figure 3).

Overall survival and progression-free survival. PFS and OS of patients treated with each protocol were estimated. The PFS was significantly better for the GCsplit than for the GCarbo group $(p=0.0404)$. PFS at 1 year for the GCsplit, GCarbo, and GC groups was 47.6\%, 22.0\%, and 20.7\%, respectively. The median PFS for the GCsplit, GCarbo, and GC groups was 9.9, 6.4, and 5.3 months, respectively (Figure 4 , left). The OS was also significantly better for the GCsplit than for the GCarbo groups ( $p=0.0454)$. OS at 1 year for the GCsplit, GCarbo, and GC groups was $80.0 \%, 55.1 \%$, and $33.9 \%$, respectively. The median OS for the GCsplit, GCarbo, and GC groups was 18.1, 12.5, and 11.2 months, respectively (Figure 4, right).

Frequency and grade of AEs. Neutropenia was significantly more severe for the GCsplit group than the GCarbo group $(p=0.0103)$. All patients treated with GCsplit had grade 3 or 4 neutropenia, and $80 \%$ of the patients had grade 4 neutropenia. Conversely, although $81 \%$ of the patients treated with GCarbo had grade 3 or 4 neutropenia, only $25 \%$ had grade 4 neutropenia (Figure 5, left). In the GCarbo group, all 

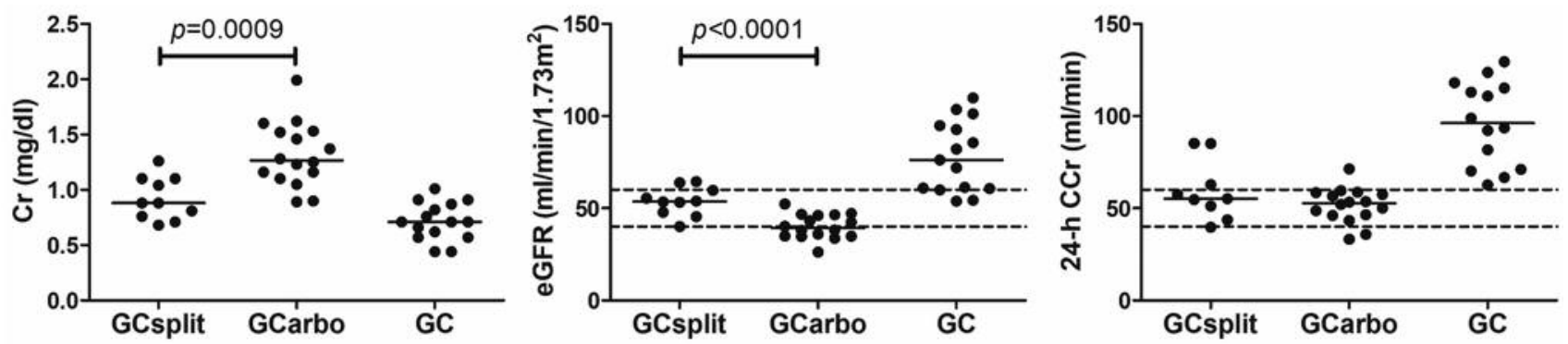

Figure 1. Serum creatinine level (Cr), estimated glomerular filtration rate (eGFR) based on Cr, and 24-h creatinine clearance (24-h CCr) with correction by body surface area are shown for patients treated with gemcitabine/cisplatin (GC), GCsplit or gemcitabine/carboplatin (GCarbo). Broken lines in eGFR and 24-h CCr graphs show rates of 40 and $60 \mathrm{ml} / \mathrm{min}$.

Table II. Characteristics of patients treated with gemcitabine/cisplatin (GC), GCsplit or gemcitabine/carboplatin (GCarbo) based on blood test data. Values are the median (range).

\begin{tabular}{lcccc}
\hline Characteristic & $\begin{array}{c}\text { GCsplit } \\
(\mathrm{n}=10)\end{array}$ & $\begin{array}{c}\text { GCarbo } \\
(\mathrm{n}=16)\end{array}$ & $\begin{array}{c}\text { GC } \\
(\mathrm{n}=15)\end{array}$ \\
\hline $\mathrm{WBC}, \times 10^{3} / \mu \mathrm{l}$ & $4.97(2.00-10.3)$ & $6.09(3.75-7.15)$ & $5.67(3.61-15.2)$ & 0.6500 \\
$\mathrm{~N} / \mathrm{L} \mathrm{ratio}$-Value $^{*}$ & $3.6(1.2-7.6)$ & $2.9(1.5-7.7)$ & $3.2(1.5-19.7)$ & 0.5721 \\
$\mathrm{Hb}, \mathrm{g} / \mathrm{dl}$ & $11.8(6.5-13.0)$ & $11.5(8.6-13.1)$ & $10.8(7.0-15.7)$ & 0.3910 \\
$\mathrm{Platelet}, \times 10^{4} / \mu \mathrm{l}$ & $16.9(10.3-50.4)$ & $22.7(14.7-34.1)$ & $26.0(14.4-76.7)$ & 0.6581 \\
$\mathrm{CRP}, \mathrm{mg} / \mathrm{dl}$ & $0.25(0-4.6)$ & $0.65(0-8.8)$ & $0.70(0.10-10.5)$ & 0.3235 \\
$\mathrm{TP}, \mathrm{g} / \mathrm{dl}$ & $6.6(4.5-7.3)$ & $6.9(6.2-8.0)$ & $6.6(5.7-7.7)$ & 0.0804 \\
$\mathrm{Alb}, \mathrm{g} / \mathrm{dl} l^{* *}$ & $3.8(1.9-4.2)$ & $3.8(2.8-4.3)$ & $14(7-22)$ & 0.4783 \\
$\mathrm{BUN}, \mathrm{mg} / \mathrm{dl}$ & $17(11-66)$ & $21.5(16-47)$ & $0.71(0.44-1.01)$ & 0.7493 \\
$\mathrm{Cr}, \mathrm{mg} / \mathrm{dl}$ & $0.88(0.68-1.26)$ & $1.27(0.89-1.99)$ & $76.2(53.8-110)$ & 0.0009 \\
$\mathrm{eGFR}, \mathrm{ml} / \mathrm{min} / 1.73 \mathrm{~m}^{2}$ & $53.6(40.1-64.4)$ & $39.2(26.2-52.3)$ & $96.2(62.7-129)$ & $<0.0001$ \\
$24-\mathrm{h} \mathrm{CCr}, \mathrm{ml} / \mathrm{min}^{* *}$ & $55.2(39.7-85.2)$ & $52.7(33.2-71.3)$ & 0.1327 \\
\hline
\end{tabular}

WBC: White blood cell, N/L ratio: neutrophil-to-lymphocyte ratio, Hb: hemoglobin, CRP: C-reactive protein, TP: total protein, Alb: albumin, BUN: Blood urea nitrogen, Cr: creatinine, eGFR: estimated glomerular filtration rate, CCr: creatinine clearance. *GCsplit $v s$. GCarbo. **Patients whose data are not available (missing data) were removed from the analyses.

patients had grade 2 or severe anemia, and one patient had grade 4 anemia. In the GCsplit group, $20 \%$ of the patients had grade 1 anemia, and none of the patients had grade 4 anemia (Figure 5, middle). There was no significant difference in the severity of anemia between the GCsplit and GCarbo groups. Regarding thrombocytopenia, the distribution of grade in both GCsplit and GCarbo groups was almost similar and not significantly different (Figure 5, right).

\section{Discussion}

Dogliotti et al. compared GCarbo with GC for patients who were cisplatin-eligible with GFR $>60 \mathrm{ml} / \mathrm{min}$ and showed equality to GC in PFS, OS, and toxicity (10). However, ORR in the GC and GCarbo groups was $65.9 \%$ and $56.4 \%$, respectively (10). Moreover, the sample size of their study was relatively small, with a total of only 110 patients (10). Even if the efficacy of GC and GCarbo are similar for patients who are cisplatin-eligible, this is still not enough to confirm GCarbo as
Table III. Response in tumor size of patients treated with gemcitabine/cisplatin (GC), GCsplit or gemcitabine/carboplatin (GCarbo). There were no significant differences in response.

\begin{tabular}{lccc}
\hline & $\begin{array}{c}\text { GCsplit, n }(\%) \\
(\mathrm{n}=10)\end{array}$ & $\begin{array}{c}\text { GCarbo, }(\%) \\
(\mathrm{n}=16)\end{array}$ & $\begin{array}{c}\mathrm{GC}, \mathrm{n}(\%) \\
(\mathrm{n}=15)\end{array}$ \\
\hline ORR & $8(80)$ & $9(56)$ & $10(67)$ \\
CR & $2(20)$ & $3(19)$ & $5(33)$ \\
PR & $6(60)$ & $6(38)$ & $5(33)$ \\
SD & $2(20)$ & $6(38)$ & $5(33)$ \\
PD & $0(0)$ & $1(7)$ & $0(0)$ \\
\hline
\end{tabular}

ORR: Overall response rate; CR: complete response; PR: partial response; SD: stable disease; PD: progressive disease.

the standard of care for patients who are cisplatin-ineligible. GCarbo was also compared with methotrexate/carboplatin/ vinblastine (M-CAVI) for patients with renal function ineligible for cisplatin with GFR $<60 \mathrm{ml} / \mathrm{min}$. It showed less toxicity and 


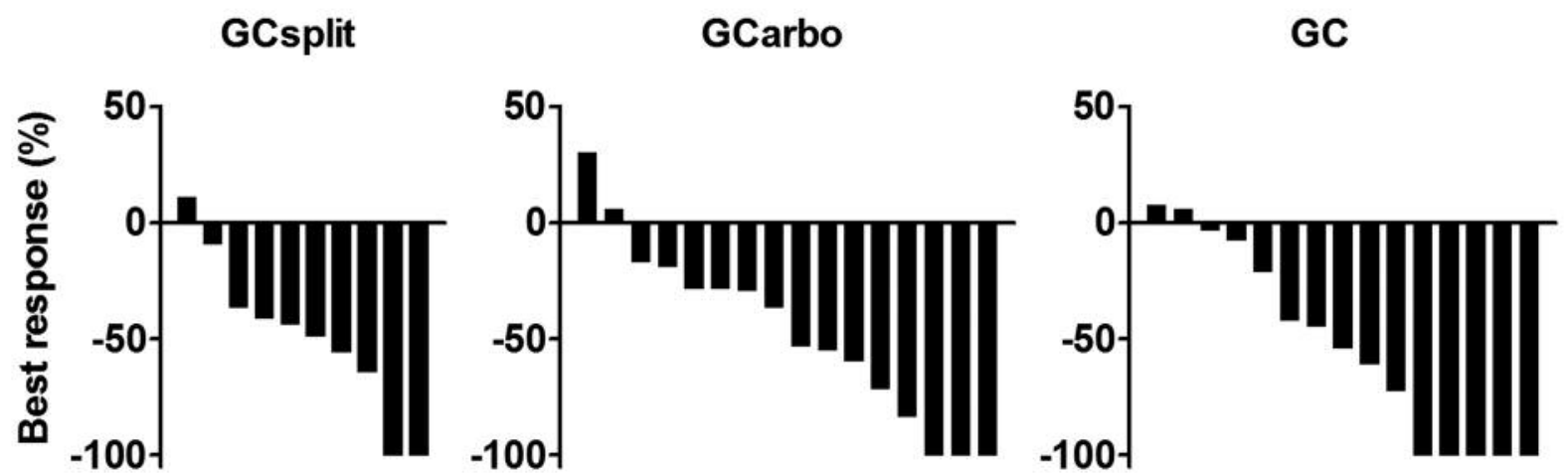

Figure 2. Waterfall plots of the best response rate compared to baseline for patients treated with gemcitabine/cisplatin (GC), GCsplit or gemcitabine/carboplatin (GCarbo).

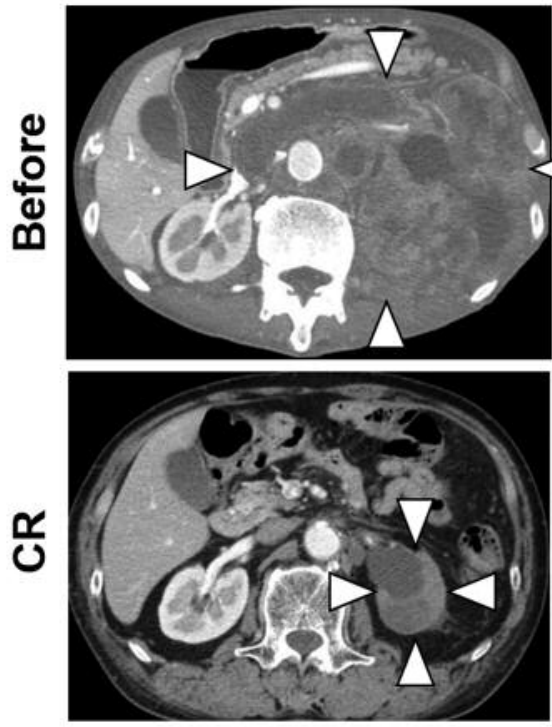

GCsplit
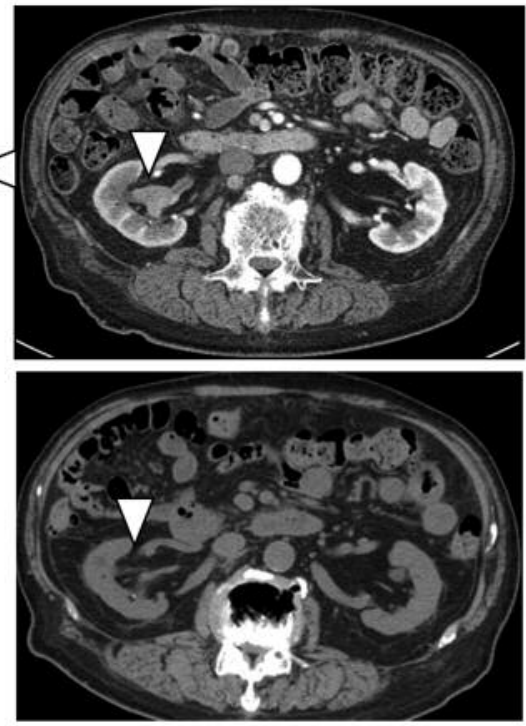

GCarbo
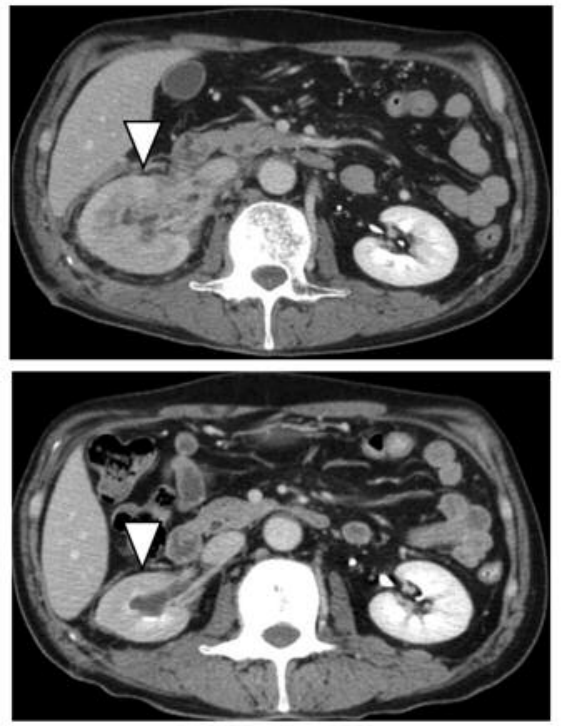

GC

Figure 3. Computed tomographic images are shown before treatment and at complete response (CR) for representative CR cases for patients treated with gemcitabine/cisplatin (GC), GCsplit or gemcitabine/carboplatin (GCarbo). White triangles indicate tumors.

equality to M-CAVI in terms of PFS and OS (6). However, MCAVI was not recognized as the standard of care. There is still no evidence regarding the efficacy of GCarbo proven by prospective randomized controlled trials for patients who are cisplatin-ineligible. As the survival time of patients with LAMUC without chemotherapy is extremely limited, it is necessary for patients, urologists, and oncologists to exploit the standard treatment for patients with LAMUC who are cisplatinineligible (11).

In the present study, GCsplit led to significantly better OS and PFS, in addition to a tendency for a better effect on tumor shrinkage than GCarbo for patients who were cisplatin-ineligible. Moreover, despite the background of patients being unfavorable, GCsplit may be even better than GC for patients who are cisplatin-eligible. For patients with LAMUC who are cisplatin-eligible, the ORR of GCarbo was $56 \%$, which is similar to that of GCarbo in a previous reported study (10). This suggests that the background of the present study may be like the study for patients who are cisplatin-eligible, and GCsplit may contribute to survival benefit even in patients who are cisplatin-eligible. This superiority regarding survival of GCsplit over GCarbo may 
PFS

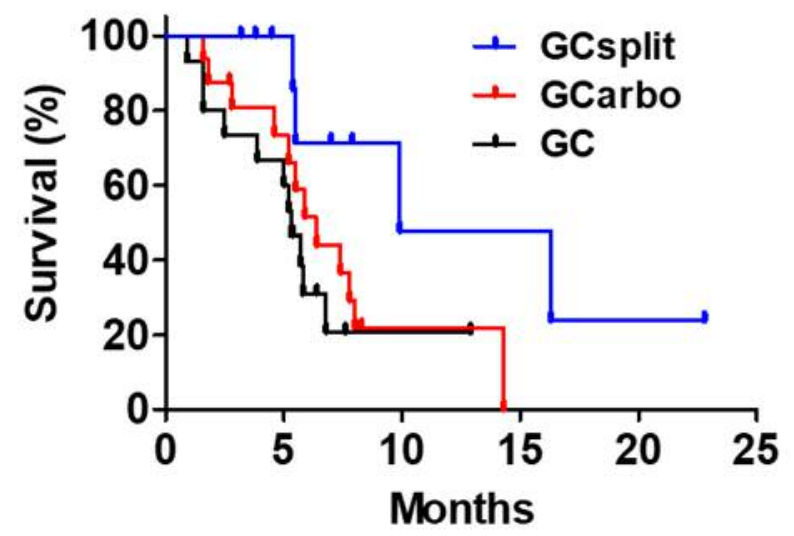

OS

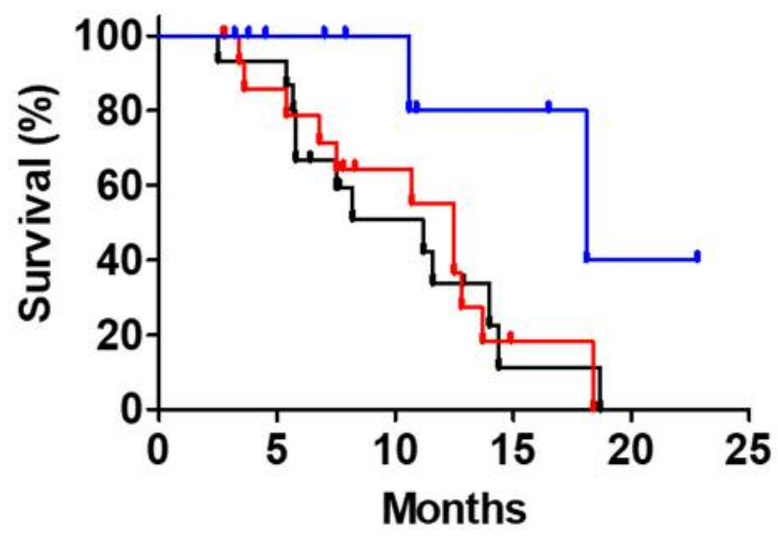

Figure 4. Patients were treated with gemcitabine/cisplatin (GC), GCsplit or gemcitabine/carboplatin (GCarbo). Progression-free survival (PFS) and overall survival (OS) of patients treated with each protocol were estimated. The Kaplan-Meier method was used to display OS and PFS, and the log-rank test was used for statistical analyses. The PFS was significantly better for the GCsplit than for the GCarbo group ( $p=0.0404)$. The OS was also significantly better for the GCsplit than for the GCarbo groups $(p=0.0454)$.
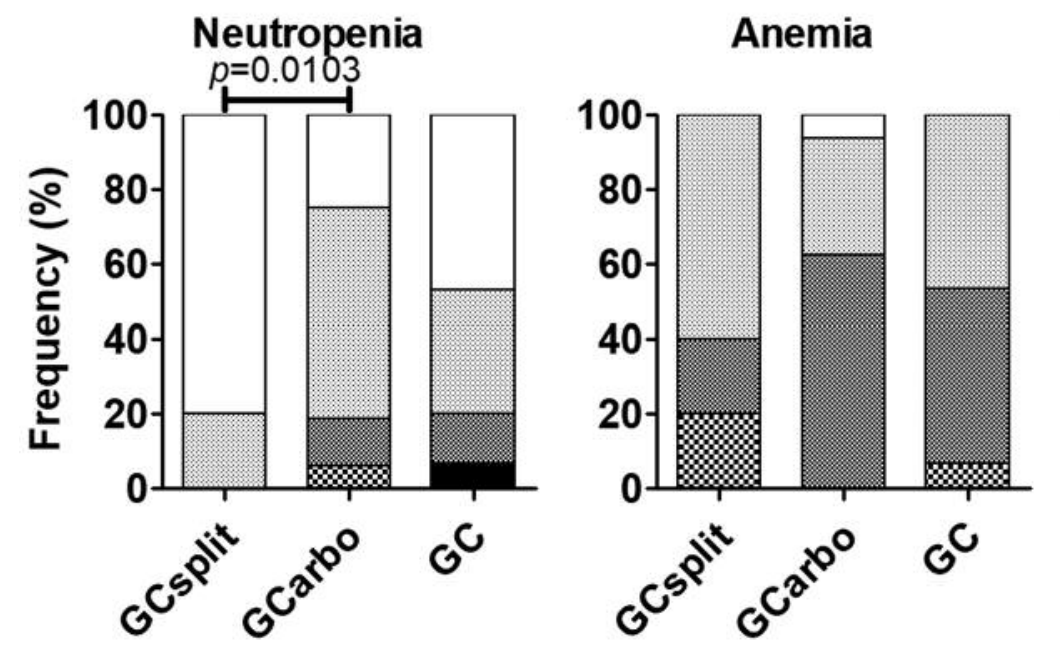

\section{Thrombocytopenia}

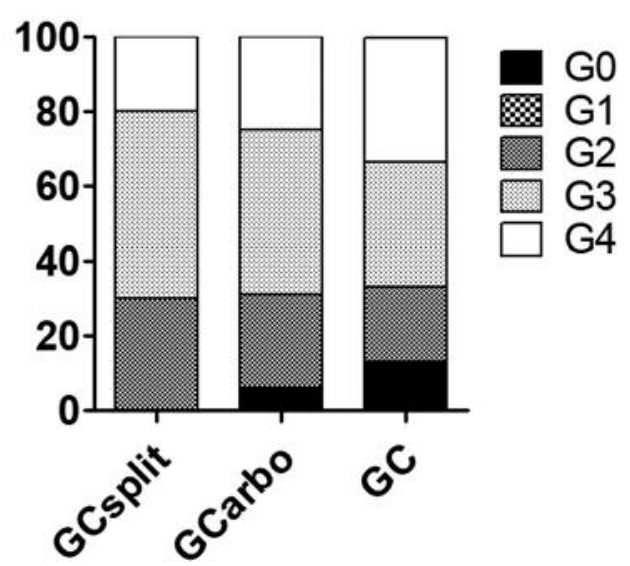

Figure 5. The frequency and grade $(G)$ of hematological adverse events for patients treated with gemcitabine/cisplatin (GC), GCsplit or gemcitabine/carboplatin (GCarbo) are shown.

be attributed to its intensity, making it strong enough for patients with unfavorable backgrounds. These data suggest that GCsplit may be more beneficial than GCarbo for patients who are cisplatin-ineligible.

Regarding AEs, neutropenia in the GCsplit group was $100 \%$ at grade 3 and 4 , which was more severe than that for the GCarbo group with $81 \%$ at grade 3 and 4 . It was reported that the frequency of grade 3 and 4 neutropenia in the GC and GCarbo groups for cisplatin-eligible patients with LAMUC was $35 \%$ and $45 \%$, respectively (10). As hematological AEs of chemotherapy were relatively more severe for Asian patients than for non-Asian patients (12), a high incidence of grade 3 and 4 AEs in the present study may be consistent with the high efficacy of GCsplit for patients who are ineligible for cisplatin therapy. There was a significant difference in neutropenia; however, the severity of thrombocytopenia and anemia between the GCsplit and GCarbo groups were similar, and these hematological AEs may be manageable. Kim et al. reported not only favorable responses but also tolerability of GCsplit compared to GCarbo (7). Combining their data and 
the results in this study, GCsplit may be a promising alternative to GCarbo for patients with LAMUC who have cisplatin-unfit renal function.

This study has certain limitations. This was a retrospective study with a rather small sample size, and all patients were Japanese. Accumulation of evidence is needed because the incidence rate of LAMUC with GFR 40-60 $\mathrm{ml} / \mathrm{min}$ is relatively low. A prospective randomized controlled trial with a largesized population is needed to confirm our preliminary findings of the efficacy of GCsplit for patients with LAMUC who are ineligible for cisplatin. However, to our knowledge, this is the first report to clarify the efficacy of GCsplit, and GCsplit may be a candidate for the first-line treatment of cisplatin-ineligible patients with LAMUC even with a high incidence of neutropenia. In conclusion, GCsplit may be a better treatment candidate for patients with advanced urothelial cancer who have cisplatin-ineligible renal function. However, a prospective randomized controlled trial with a large-sized population is needed to confirm our preliminary results.

\section{Conflicts of Interest}

The Authors declare that they have no conflicts of interest in regard to this study.

\section{References}

1 Izumi K, Itai S, Takahashi Y, Takahashi R, Maolake A, Ofude M, Ueno S, Kadono Y, Kitagawa Y, Konaka H, Mizokami A and Namiki M: Factors predictive of oncological outcome after nephroureterectomy: Comparison between laparoscopic and open procedures. Anticancer Res 33: 5501-5506, 2013.

2 Iwamoto H, Izumi K, Shimura Y, Natsagdorj A, Maolake A, Takezawa Y, Nohara T, Shigehara K, Kadono Y and Mizokami A: Metastasectomy improves survival in patients with metastatic urothelial carcinoma. Anticancer Res 36: 5557-5561, 2016.

3 Loehrer PJ, Sr., Einhorn LH, Elson PJ, Crawford ED, Kuebler P, Tannock I, Raghavan D, Stuart-Harris R, Sarosdy MF and Lowe BA: A randomized comparison of cisplatin alone or in combination with methotrexate, vinblastine, and doxorubicin in patients with metastatic urothelial carcinoma: A Cooperative Group study. J Clin Oncol 10: 1066-1073, 1992.

4 von der Maase H, Sengelov L, Roberts JT, Ricci S, Dogliotti L, Oliver T, Moore MJ, Zimmermann A and Arning M: Long-term survival results of a randomized trial comparing gemcitabine plus cisplatin, with methotrexate, vinblastine, doxorubicin, plus cisplatin in patients with bladder cancer. J Clin Oncol 23: 46024608, 2005.
5 Galsky MD, Hahn NM, Rosenberg J, Sonpavde G, Hutson T, Oh WK, Dreicer R, Vogelzang N, Sternberg CN, Bajorin DF and Bellmunt J: Treatment of patients with metastatic urothelial cancer "unfit" for cisplatin-based chemotherapy. J Clin Oncol 29: 2432-2438, 2011.

6 De Santis M, Bellmunt J, Mead G, Kerst JM, Leahy M, Maroto P, Gil T, Marreaud S, Daugaard G, Skoneczna I, Collette S, Lorent J, de Wit R and Sylvester R: Randomized phase II/III trial assessing gemcitabine/carboplatin and methotrexate/ carboplatin/vinblastine in patients with advanced urothelial cancer who are unfit for cisplatin-based chemotherapy: EORTC study 30986. J Clin Oncol 30: 191-199, 2012.

7 Kim YR, Lee JL, You D, Jeong IG, Song C, Hong B, Hong JH and Ahn H: Gemcitabine plus split-dose cisplatin could be a promising alternative to gemcitabine plus carboplatin for cisplatin-unfit patients with advanced urothelial carcinoma. Cancer Chemother Pharmacol 76: 141-153, 2015.

8 Common Terminology Criteria for Adverse Events (CTCAE) v4.0. https://ctep.cancer.gov/protocolDevelopment/electronic_ applications/ctc.htm\#ctc_40

9 Eisenhauer EA, Therasse P, Bogaerts J, Schwartz LH, Sargent D, Ford R, Dancey J, Arbuck S, Gwyther S, Mooney M, Rubinstein L, Shankar L, Dodd L, Kaplan R, Lacombe D and Verweij J: New response evaluation criteria in solid tumours: revised RECIST guideline (version 1.1). Eur J Cancer 45: 228-247, 2009.

10 Dogliotti L, Carteni G, Siena S, Bertetto O, Martoni A, Bono A, Amadori D, Onat $\mathrm{H}$ and Marini L: Gemcitabine plus cisplatin versus gemcitabine plus carboplatin as first-line chemotherapy in advanced transitional cell carcinoma of the urothelium: Results of a randomized phase 2 trial. Eur Urol 52: 134-141, 2007.

11 Saxman SB, Propert KJ, Einhorn LH, Crawford ED, Tannock I, Raghavan D, Loehrer PJ Sr, Trump D: Long-term follow-up of a phase III intergroup study of cisplatin alone or in combination with methotrexate, vinblastine, and doxorubicin in patients with metastatic urothelial carcinoma: a Cooperative Group study. J Clin Oncol 15: 2564-2569, 1997.

12 Yano R, Konno A, Watanabe K, Tsukamoto H, Kayano Y, Ohnaka H, Goto N, Nakamura T and Masada M: Pharmacoethnicity of docetaxel-induced severe neutropenia: integrated analysis of published phase II and III trials. Int J Clin Oncol 18: 96-104, 2013.

Received October 12, 2018

Revised October 29, 2018 Accepted November 1, 2018 